\title{
Real-Time User Localisation in Beam Steered NIR Optical Wireless Communications
}

\author{
Chaitanya K. Mididoddi ${ }^{1}$, Guoqing Wang ${ }^{1}$, Xiaojuan Liu ${ }^{1,2}$, and Chao Wang, ${ }^{1, *}$ \\ ${ }^{1}$ School of Engineering and Digital Arts, University of Kent, Canterbury, United Kingdom, CT2 7NT \\ ${ }^{2}$ School of Physics and Optoelectronics Engineering, Shandong University of Technology, Zibo, China, 255049 \\ *e-mail: c.wang@kent.ac.uk
}

\begin{abstract}
Near infrared (NIR) optical wireless communication provides a promising solution for point-to-point indoor high speed wireless data link. To cover a large area and several multiple users, wavelength-encoded laser beam steering has been demonstrated in previous research work. One remaining challenge in beam steered optical wireless system is real-time user localization. In this paper, ultrafast complete user localization at update rate of $10 \mathrm{MHz}$ based on instantaneous optical wavelength detection and chirped pulse correlation has been demonstrated. Both angular position and absolute distance of each user have been accurately detected.
\end{abstract}

Index Terms - beam steering, chirp, dispersion, near infrared, optical wireless communication, photonic time stretch, tilted Fibre Bragg grating

\section{INTRODUCTION}

As a promising alternative option to visible light communications (VLC), near infrared (NIR) lasers have attracted great interest in indoor optical wireless communications. Unique advantages offered by NIR optical wireless communications include readily available devices originated from optical fibre communication systems, seamless interface with the existing fibre-to-home (FTTH) access networks, unshared data transmission for each individual user, higher link power budget and higher photodetection sensitivity in NIR. Due to the fact that pencil laser beam is used, beam steering is essentially required to cover large area and serve multiple users or track mobile users. To reduce the system complexity and cost in active mechanical beam steering solutions and improve beam steering speed, mechanical-free and passive laser beam steering has been demonstrated for indoor optical wireless communications [1], which is made possible based on wavelength-encoded beam scanning using diffraction device [2]. All fibre beam diffraction has also been demonstrated based on the use of an in-fibre diffraction grating [3] for full duplex optical wireless transmission [4]. Compared to conventional free-space diffraction gratings which suffer from limited diffraction efficiency, high insertion loss, and bulky footprint, in-fibre diffraction device based on a $45^{\circ}$ tilted fibre grating (TFG) offers compactness, inherent compatibility with optical fibres and greatly improved diffraction efficiency [3].

One remain challenge in beam steered optical wireless communications is real-time and accurate user localization for multiple user scenarios with high mobility. Existing indoor user localization approaches based on WiFi, Bluetooth, and
UWB [5] need extra hardware to existing optical wireless communication systems and more importantly suffer from limited positioning resolution, which is however of paramount importance to wavelength-controlled beam steering systems $[1,4]$. In this paper, we propose and demonstrate a new realtime and accurate user localization approach which is inherently compatible to the wavelength-controlled beam steered NIR optical wireless communication setup. In the system, an ultrafast passive wavelength sweeping module based on dispersive Fourier transform [6] is used to determine the angular position of the users [7] and wavelength-independent time-offlight approach based on pulse cross-correlation is used to estimate the absolute distance between the users and the access point. Therefore, complete user localization can be achieved with ultrafast update rate of tens of $\mathrm{MHz}$.

\section{PRINCIPLE}

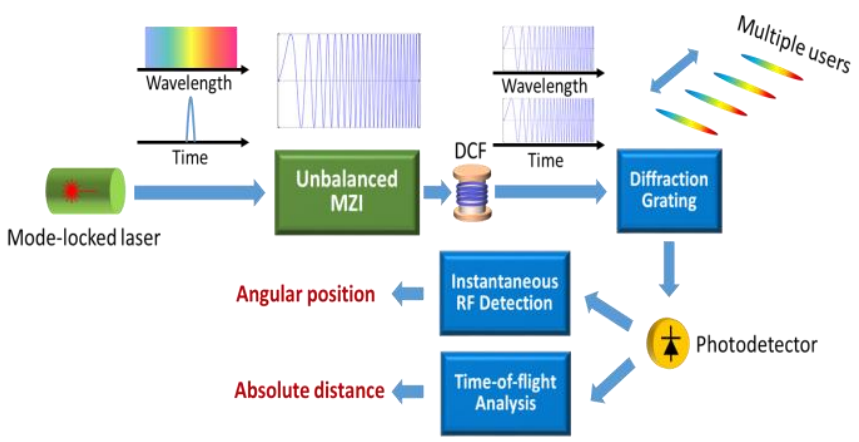

Fig. 1. Schematic diagram of the proposed real-time user localization system based on instantaneous optical wavelength detection for angular position and time-of-the-flight for absolute distance measurement.

Figure 1 shows the schematic diagram of the proposed ultrafast beam scanning based user localization system. A modelocked fibre laser (MLL) generates ultrafast optical pulses with broad spectrum. A dispersion unbalanced Mach-Zehnder interferometer (MZI) serves as an optical spectral shaper. One arm of the MZI has a length of dispersive optical fibre and the other arm provides a tunable optical delay. Therefore, the dispersion unbalanced MZI has a chirped spectral response [8]. After time stretched by a dispersion compensating fibre (DCF), the dispersed optical pulse has a chirped microwave 
modulation frequency profile thanks to dispersion-induced wavelength-to-time mapping. The central frequency is determined by the optical delay in MZI and the frequency chirp rate is determined by the dispersion difference between two arms of the MZI. As a result, the instantaneous optical wavelength within the time stretch optical pulse can be identified by detecting the instantaneous microwave carrier frequency [7].

When the dispersed optical pulse is emitted from a diffraction device, such as a free-space diffraction grating [1] or an in-fibre $45^{\circ}$ tilted fibre grating (TFG) [4], ultrafast laser beam scanning is achieved due to wavelength to angle (space) mapping at the diffraction grating device. The scanning speed is same as the pulse repetition rate, which can be $\mathrm{MHz}$, even in $\mathrm{GHz}$ range. The specific optical wavelength that serves a particular user can be detected in real time by measuring the instantaneous microwave frequency of the reflected narrow band pulse from the user using short-time Fourier transform method. Therefore, the angular position of the user can be determined via the wavelength-angle conversion map [7].

The absolute distance between the user and the fibre access point needs to be measured as well to determine the complete position of the user. In this paper, we propose to use cross correlation between the pre-recorded time stretch optical pulse and the user-reflected narrow band pulse to determine the time-of-flight, hence the absolute distance. Note that the direct cross correlation results include not only the actual time of flight, but also the wavelength-dependent time delay within the time stretched optical pulse. The latter can be estimated by knowing the instantaneous optical wavelength and the total dispersion value.

\section{RESULTS}

Utilizing photonic time stretch based ultrafast laser beam steering and frequency chirping enabled instantaneous optical wavelength detection, as well as cross correlation based timeof-flight measurement, numerical simulations are carried out using commercial software package VPItranmissionMaker to verify the proposed approach assuming parameters used in our previous experimental systems. Ultrafast optical pulses with full-width half maximum pulse width of $500 \mathrm{fs}$ and repetition rate of $10 \mathrm{MHz}$ are produced by a passively mode-locked laser. The unbalanced MZI has a dispersion difference of between two arms, which generates a chirped spectral response and is used to filter the pulse spectrum. The spectrally shaped pulses are then stretched by a DCF device with total dispersion value of $1280 \mathrm{ps} / \mathrm{hm}$, leading to a frequency chirped waveform, as shown in Fig. 2(a). The linear instantaneous carrier frequency is calculated based on short-time Fourier transform and the result is shown in Fig. 2(b). The time stretched and frequency chirped optical pulses enable ultrafast laser beam scanning with the help of a diffraction grating device.
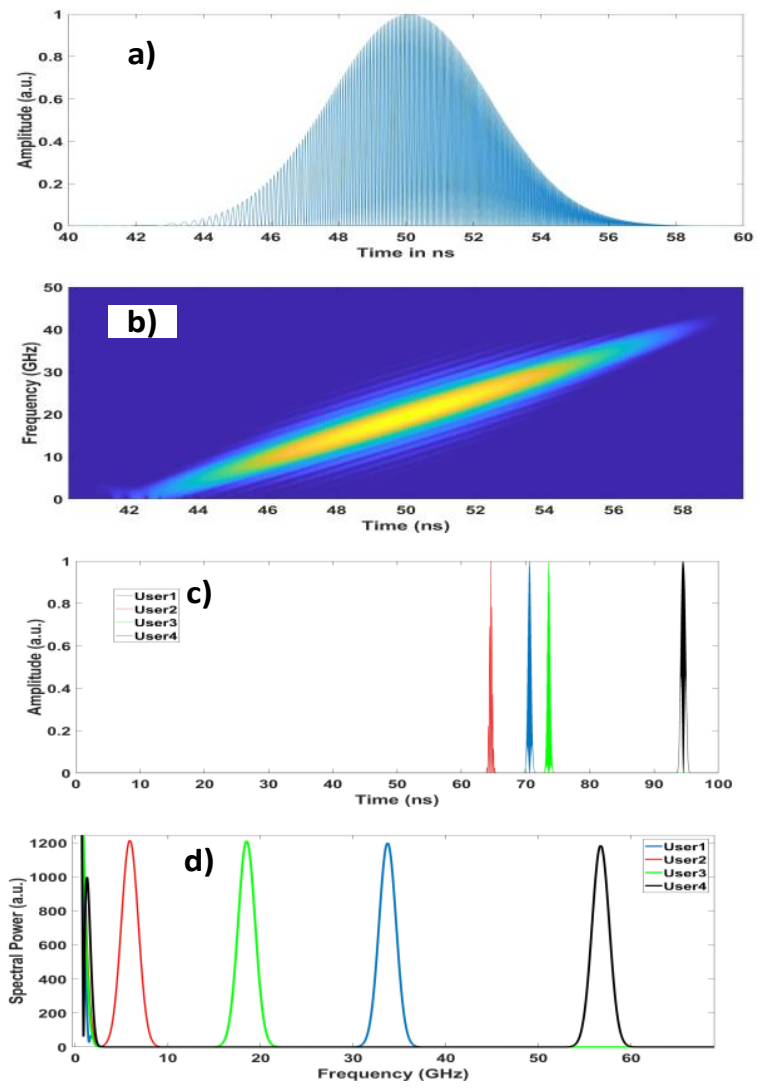

Fig. 2. Results for angular position measurement based on instantaneous microwave frequency detection. (a) Time stretched optical pulse after unbalanced MZI filter and dispersion stretch with linear frequency chirp; (b) spectrogram showing linearly increasing instantaneous microwave frequency across the pulse duration; (c) reflected pulses from four users with each carrying different chirp frequencies; (d) instantaneous calculated for the four pulses using short-time Fourier transform, which can be used to determine the optical wavelength for different users.

Here four users served by four different optical wavelengths (user 1: $194.1 \mathrm{THz}$, user 2: $193 \mathrm{THz}$, user 3: $193.5 \mathrm{THz}$ and user 4: $195 \mathrm{THz}$ ) and with different distances to the fibre access point (user 1: $4.5 \mathrm{~m}$, user 2: $6 \mathrm{~m}$, user $3: 7.2 \mathrm{~m}$ and user 4 : $9 \mathrm{~m})$ are considered. Figure 2(c) shows the reflected pulses from the four users. Note that since each user is allocated with a unique optical wavelength and the total channel bandwidth is $50 \mathrm{GHz}$ [7], therefore the reflected pulses are much shorter than the whole stretched pulse in Fig. 2(a). Measuring the instantaneous optical wavelength for each narrow-band pulse is very challenging. Here each pulse has been encoded with different chirped microwave modulation frequency. Therefore, the instantaneous microwave frequency can be calculated using short-time Fourier with results shown in Fig. 2(d). The unknown instantaneous optical wavelengths serving the four users can then be determined based on two linear mapping relations: instantaneous RF frequency and time, instantaneous optical wavelength (frequency) and time, as shown in Fig. 3. Therefore, the optical wavelengths serving the users and hence 
the angular positions of the users can be uniquely determined due to wavelength-to-angle mapping at the diffraction grating.
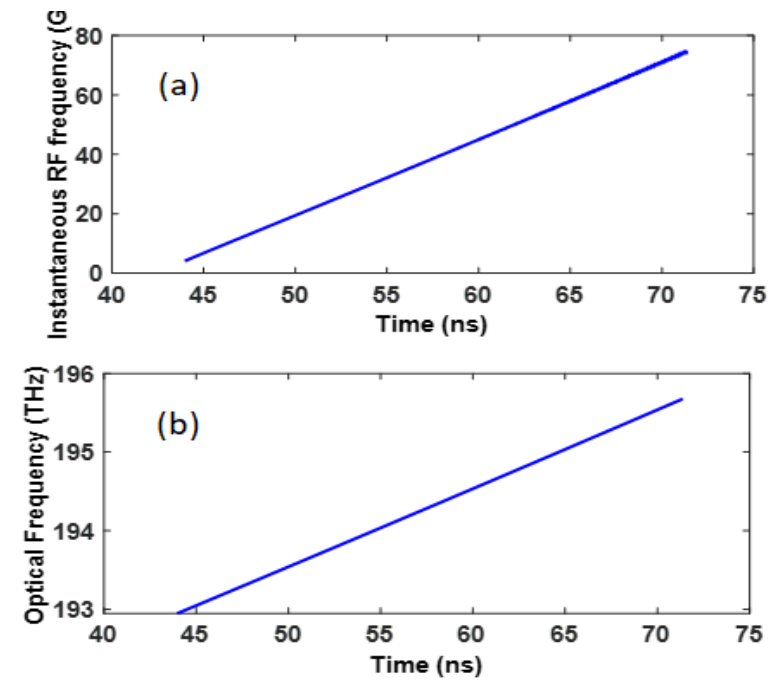

Fig. 3. Linear relations in photonic time stretch and frequency chirping. (a) calculated instantaneous RF frequency within the stretch optical pulse showing perfect linear RF frequency to time mapping; (b) direct optical frequency to time mapping thanks to photonic time stretch.

Absolute distances from the fibre access point to all users need to be measured as well to form a complete user localization. Time-of-flight is a simple and straightforward solution. However, the reflected narrow-band pulses from the users are delayed not only because the propagation in free space, but also the wavelength-dependent time delay within the original chirped optical pulse. As shown in Fig. 2(c), the reflected pulses from four users have time delays of $70.67 \mathrm{~ns}, 64.35 \mathrm{~ns}$, $73.50 \mathrm{~ns}$ and $94.62 \mathrm{~ns}$, respectively. Removing the initial wavelength-dependent time delays, which can be determined based on the detected optical wavelength and the linear relation in time stretch process as shown in Fig. 3(b), the actual time-of-flight values for the four users are estimated as 15.006 $\mathrm{ns}, 20.005 \mathrm{~ns}, 24.001 \mathrm{~ns}$ and $30.004 \mathrm{~ns}$, corresponding to absolute distances of $4.502 \mathrm{~m}, 6.002 \mathrm{~m}, 7.2 \mathrm{~m}$ and $9.001 \mathrm{~m}$, respectively, which are in good agreement with the preset values.

\section{CONCLUSION}

Ultrafast laser beam scanning based on photonic time stretch of ultrafast optical pulses and spatial diffraction offers a promising solution for real time user localization in NIR beam steered optical wireless communications. In this paper we proposed and demonstrated that the angular position of a user can be determined by optical wavelength detection via instantaneous microwave modulation frequency measurement and the absolute distance between fibre access point and the user can be estimated based on time-of-flight taking into account the wavelength-dependent time delay in stretched optic pulse. The proposed real-time localization approach provides great promise in NIR beam steered optical wireless communications with unique features of high speed, inherent compatibility with existing beam steering system, and complete positioning.

\section{ACKNOWLEDGMENT}

This work was supported in part by the EU FP7 MarieCurie Career Integration Grant (631883) and in part by the Royal Society (IE170007).

\section{REFERENCES}

[1] C. W. Oh, Z. Cao, E. Tangdiongga, and T. Koonen, "Free-space transmission with passive 2D beam steering for multi-gigabit-per-second perbeam indoor optical wireless networks," Opt. Express, vol. 24, no. 17, pp. 19211-19227, Aug. 2016.

[2] K. Goda, A. Mahjoubfar, C. Wang, A. Fard, J. Adam, D. R. Gossett, A. Ayazi, E. Sollier, O. Malik, E. Chen, Y. Liu, R. Brown, N. Sarkhosh, D. Di Carlo and B. Jalali, "Hybrid dispersion laser scanner," Sci. Rep., vol. 2, pp. 445, Jun. 2012.

[3] G. Wang, Z. Yan, L. Yang, L. Zhang, and C. Wang, "Improved resolution optical time stretch imaging based on high efficiency in-fiber diffraction," Sci. Rep., vol. 8, no. 1, p. 600, Jan. 2018.

[4] G. Wang, U. Habib, Z. Yan, N. J. Gomes, Q. Sui, J. Wang, L. Zhang, and C. Wang, "Highly efficient optical beam steering using an in-fiber diffraction grating for full duplex indoor optical wireless communication," J. Lightwave Technol., vol. 36, no. 19, pp. 4618-4625, Oct. 2018.

[5] C. Yang and H. Shao, "WiFi-based indoor positioning," IEEE Commun. Mag., vol. 53, no. 3, pp. 150-157, 2015.

[6] C. Wang, "Dispersive Fourier transformation for versatile microwave photonics applications," Photonics, vol. 1, no. 4, pp. 586-612, Dec 2014.

[7] C. K. Mididoddi, G. Wang, U. Habib, H. Zhang, and C. Wang, "Ultrafast user localization and beam steering in optical wireless communication using an in-fibre diffraction grating," in 2018 International Topical Meeting on Microwave Photonics (MWP), 2018, pp. 1-4.

[8] W. Liu, M. Li, C. Wang, and J. Yao, "Real-time interrogation of a linearly chirped fiber Bragg grating sensor based on chirped pulse compression with improved resolution and signal-to-noise ratio," J. Lightwave Technol., vol. 29, no. 9, pp. 1239-1247, 2011. 